\title{
Using CATI in survey research by telephone
}

Various methods in survey research take advantage of computer as a tool for data collection. Computer-assisted personal interviewing (CAPI), telephone interviewing (CATI) as well as self-interviewing (CASI) use a computerized questionnaire as an efficient replacement of paper and pencil. This paper, however, focuses on the advantages of computer-assisted telephone surveys and points out potential weaknesses.

Most CATI systems operate from centralized telephone laboratories equipped with a server, a supervisor workstation and several interviewer stations; these are connected as a local area network (LAN). The field work is managed by a CATI software, which can be a self-developed solution or found on a large market of third-party software for almost any computer architecture (see web links). Setting-up a CATI network does not require the latest hardware. A network consisting of standard work stations and server can adequately run most CATI software. Additional hardware, such as modems for automated, or a telephone system for predictive dialling, is optional. The features of a CATI system depend on the software used. But some basic functions should be part of all systems (Tab. 1).

One principal function of CATI software is questionnaire design. Depending on the software package, these are programmed with macro or scripting languages or written with a graphical user interfaces using pre-designed question templates, which requires no special programming skills.

The advantages of computerized questionnaire are obvious: Questions are read from the screen and respondent's answers typed in directly; time-consuming and error prone coding is not necessary; filters, skips, loops and the routing through the questionnaire are managed by the software, and the interviewer can concentrate on questions and answers. Moreover, the computerised questionnaire enables range checks and cross checks for plausible answer patterns. While skip-instructions in paper-and-pencil interviewing are lim- ited, as they must immediately follow the determining answer ("if yes, skip to question 5"), computerized questionnaires allow even very complex skip-instructions ("If male, age $<60$, full time job, married, and answer to question 25 equals 1 , skip to question..."). Moreover, the course of

Table 1 Checklist for CATI evaluation and software needs

General

$\checkmark$ Pricing and licence policy: yearly or one time licence fee, open source software

$\checkmark$ Scalability: maximum number of interviewing station, upgrading and add-on modules

$\checkmark$ Supported operating system and network layouts

$\checkmark$ Call management: automated dialling, predictive dialling option

$\checkmark$ Management of multiple studies

$\checkmark$ Management of multilingual studies

Questionnaire authoring

$\checkmark$ Kind of questionnaire writing: programming, scripting/macro language, graphical user interface

$\checkmark$ Supported question types: closed, numeric, open-ended, lists, analog scales, dates

$\checkmark$ Computational capabilities and randomisation of answer choices and questions

$\checkmark$ Implementation of skips, range checks, loops grids

$\checkmark$ Integration of external databases/programs

$\checkmark$ Number of possible variables/questions

$\checkmark$ Screen design: using fonts and colours

Sample database and interview data

$\checkmark$ Sample database and interview data import and export formats

$\checkmark$ Supports generation of RDD samples

$\checkmark$ Maximum number of sample records, user defined fields

$\checkmark$ Call management: support different time zones/daylight savings

$\checkmark$ Searching and sorting sample database

$\checkmark$ Retrieving records in case of in-bound calls

$\checkmark$ Refusal tracking

$\checkmark$ Restart of mid-terminated interviews

$\checkmark$ Recording and reporting of call outcomes (date, time, interviewer, length, result)

$\checkmark$ Definition of quota and stratification characteristics

$\checkmark$ Call-back scheduling and management

$\checkmark$ Randomised sample release

$\checkmark$ Definition of rules for sample allocation (e.g., setting up priorities for call-backs, no answers, initial refusals)

$\checkmark$ Interviewer management: defining interviewer skills

$\checkmark$ Report generation: frequencies and cross-tabulations of interview data, response and refusal rates, interviewer productivity and rating 
the interview can be influenced by the processing of external data (e.g., data from previous interviews in panel studies). Another feature of a programmed questionnaire is computation and randomisation in the course of the interview. When, for example, weight and height have been stated, computation of the body mass index can follow immediately and, as a result, respondent classified as obese can be asked additional questions. Quite obviously, more complex computations are also feasible.

Ordering effects can be minimized by randomisation of answer choices, questions or whole question blocks and, besides, there is also the possibility to implement a Kish grid for random respondent selection.

Many CATI software allows the integration of external databases, which enables online coding of standard classifications such as the International Classification of Diseases (ICD-10) or the International Standard Classification of Occupations (ISCO). Nevertheless, in order to obtain valid results, complex online coding needs specially trained interviewers.

There are many details making interviewing with computer support easier and smoother for both, the interviewer and respondent: wording of questions can be adapted for every single respondent, gender-specific phrases created and hardto-read question constructions such as "When did you begin your apprenticeship/studies/training period/employment; Interviewer: read applicable type" avoided, when the respective activity has been stated before.

The computerised questionnaire allows writing questionnaires that are "tailored" for the respondent. But all features must be carefully contemplated by the questionnaire designer; once in use, the computer consequently executes all instructions. The paper-and-pencil interviewer, for example, can decide to accept an atypical answer or answer pattern. But the computer rejects such "fuzzy" decision.

Contrary to what is often advertised, trying to modify a questionnaire once the fieldwork has started, may lead to serious methodological problems: changing a questionnaire, e.g., deleting or adding questions, or even change of wording can have biasing effects. Whenever a change becomes necessary, it must be duly documented and dated.

Most of the characteristics mentioned so far apply also to CASI and CAPI interviewing. There is, however, another principal feature specific to CATI software: sample and contact management. Prior to the start of a study, general parameters can be defined. These are for example, the maximum number of attempts to reach a potential respondent, quotas and stratification characteristics, or the set-up of RDD samples.

Computer-assisted telephone interviewing is a fast method to collect large data sets. But no matter how fast it may be and regardless of some advertising promises ("ready to analyse data in a few days"), fieldwork limited to only a few days can result in considerable no-contact rates and consequently in a selection bias. These can be avoided by defining a sufficient number of contacts on different days and day times.

Once the study characteristics are set up, the software releases sample records, administers appointments, and saves the interview data. Sample records are released randomly, with exceptions such as appointments, which are sent on due dates to the interviewer or busys, which are re-sent within a short period of time. With support of a predictive dialling system dialling is made and call outcomes are detected automatically. All calls answered "live" are passed by the dialler to an available interviewer. This technique improves efficiency and prevents bias by the interviewer in respondent selection.

Most CATI software packages store interview data and export them in binary proprietary or database format. Due to the transience of these formats, a major software revision or a file damage can result in data loss. To avoid such problems, it is important to save data, data description and questionnaires in a plain file format like XML.

Regardless of all technical skills programmed questionnaires and CATI software may provide, questionnaire construction and a careful field work remain the basic priority. In addition, the CATI cannot solve basic methodological problems of telephone surveys such as insufficient telephone coverage or the limited duration of interviews. But if a telephone survey is basically feasible, CATI facilitates data collection and helps to improve its quality.

Web links for survey research software including CATI

1. www.quirks.com/software/catisearch.asp (comparison of some software features and prices)

2. ww.aboutsoftwareforbusiness.com/directory/surveys.html

3. Directories of www.google, www.dmoz, www.searchalot (go to "Computers/Software/Marketing/Surveys/")

4. www.researchinfo.com/docs/software/index.cfm (downloadable demo software)

\section{Address for correspondence}

Steffen Niemann, M.A.

Department of Social and Preventive Medicine

Division of Social and Behavioural Health Research

Niesenweg 6

CH-3012 Bern

Tel +4131 631 3504

Fax +41316313430

e-mail: niemann@ispm.unibe.ch 